\title{
Are the same genes responsible for intra- and interspecific variability for sex comb tooth number in Drosophila?
}

\author{
SERGEY V. NUZHDIN* \& SARAH G. REIWITCH \\ Section of Evolution and Ecology, University of California at Davis, Davis, CA 95616
}

\begin{abstract}
The identification of genes contributing to speciation has the potential to provide important insights into the mechanisms of evolution. One of the most interesting unresolved puzzles is the relationship between intraspecific variability in morphological traits and their interspecific divergence. Intraspecific polymorphisms without major detrimental side-effects may serve as a substrate for selection response during speciation. The same quantitative trait loci (QTLs) may, then, account for the trait variability both within and between species. In contrast, the vast majority of intraspecific variants could be deleterious mutations that have not yet been selected out. In this case intraspecific variation would not ultimately generate interspecific trait differences. In previous work, QTLs responsible for morphological differences between animal species, including those for the secondary sexual trait sex comb tooth number, have been mapped with the resolution of chromosome segments. Here, we mapped QTLs for which alleles segregated between two laboratory lines of Drosophila melanogaster. The two QTLs identified mapped to the X chromosome and accounted for only $8 \%$ of the betweenline differences, implying that a large number of small-effect genes modify sex combs. One intraspecific QTL mapped to the same interval as the QTL for interspecific differences between $D$. simulans and D. mauritiana. Whether or not these effects result from the same genes requires further examination.
\end{abstract}

Keywords: Drosophila, QTL, sex comb.

\section{Introduction}

In the last decade, there has been renewed interest in the genetics of speciation, yielding a number of new models and empirical results. Three major directions have emerged. The first direction deals with the causes of speciation. It addresses the question how a species can split into isolated lineages as it diversifies (Kondrashov \& Kondrashov, 1999). The second goal is to understand the mechanisms of morphological divergence. The question is how in principle can morphological characters and behaviour be changed (see for example Rogers et al., 1995). The third direction is to infer actual genetics of the differences between species. The question addressed is how do genes change during and after speciation (see for review Coyne \& Orr, 1998). Ideally, these directions will merge together when it is understood which changes in gene expression lead to modifications of biochemical and developmental pathways during speciation.

*Correspondence. E-mail: svnuzhdin@ucdavis.edu

(C) 2000 The Genetical Society of Great Britain.
Most experimental data have so far been obtained about genes responsible for interspecific postzygotic isolation. For morphological and behavioural differences between species, however, chromosomes or their segments were identified in far fewer studies (a total of 38 studies are reviewed by Coyne \& Orr, 1998) and resolution at the gene level has not been achieved. In contrast, developmental biologists have found many genes which, when mutated, change morphology of the traits exhibiting interspecific differences (see for instance Carroll, 1995). The role of these genes in causing morphological differences during speciation has not yet been tested directly. The identification of genes responsible for interspecific differences in morphology has, thus, the potential to provide important insights into the mechanisms of morphological divergence attained during speciation (True et al., 1997). Sex comb tooth number is a good trait for such a study for several reasons.

Sex combs are a secondary sexual trait in Sophophora. Spieth (1952) observed a relationship between the behaviour used by males of different species attempting copulation and the presence of sex combs. 
Males with sex combs attempt intromission before mounting is complete, but males of species without sex combs do not. Using this distinction, Spieth speculated that the combs are necessary in precopulatory manoeuvring by males. In Drosophila melanogaster, sex combs are used by males during both courtship and mounting. Copulatory success for males whose sex combs were surgically removed was reduced (Spieth, 1952; Coyne, 1985). Selection on tooth number has also been documented in nature. Mating wild D. simulans males had significantly fewer teeth than did males not found copulating (Markow et al., 1996). Because the number of sex comb teeth affects mating, and the preferences of females for this structure may vary, the number of teeth and their positioning are perhaps under sexual selection. This selection should cause rapid changes in sex comb morphology and correlated changes in mating behaviour (Carson \& Lander, 1984). Supporting this view, sex combs strongly differ between races and species in the number of teeth per row, in the number of rows, and in the positions and orientation of rows. This makes sex comb structure one of the most reliable and precise keys for species classification.

The genetics of sex combs has been studied in species of the D. melanogaster complex. Sex combs are comprised of a single row of teeth in D. simulans and D. mauritiana with mean tooth numbers of 10.12 and 13.93, respectively. Coyne (1985) showed that this interspecific difference is coded by at least five quantitative trait loci (QTLs), a minimum of one on the $\mathrm{X}$ chromosome and one on both arms of each large autosome. The refined analysis of True et al. (1997) revealed two QTLs on the third chromosome. The first QTL mapped between markers tra (cytological position $73 \mathrm{~A}$ on the D. melanogaster map) and Antp (84BD); the second mapped between Ald (97B) and janA (99D) on the third chromosome. The first QTL explained $+53.6 \%$, and the second QTL $-21.5 \%$ of the interspecific difference $(+$ stands for right, and - stands for wrong QTL effects in comparison with the direction of interspecific differences). Two additional weaker QTLs were suggested, mapping to the interval between markers $v(10 \mathrm{~A})$ and run (19E) of the $\mathrm{X}$ chromosome, and near the marker twi (59C) of the second chromosome. The interspecific difference in sex comb is, thus, controlled by a genetically tractable number of QTLs (True et al., 1997).

Sex comb tooth number varies within species. In different lines of $D$. melanogaster, sex comb tooth number was 10.17, 10.69, 10.72, and 10.73 (Choi et al., 1994) with a significant line component of variance $(P<0.0004$, our reanalysis $)$. These intraspecific polymorphisms may have served as a substrate for the selection response during speciation. If this hypothesis is correct, the same polymorphisms should be responsible for the trait variability both within and between species. Alternatively, the vast majority of intraspecific variants could be deleterious mutations that have not yet been selected out. In this case intraspecific variation does not ultimately generate interspecific trait difference during speciation. No data are available about the genetics of intraspecific variability in the sex comb tooth number. Here, we identify QTLs responsible for intraspecific variability in the trait and compare them with the QTLs responsible for interspecific differences.

\section{Materials and methods}

The number of sex comb teeth was scored on each foreleg of 40 males of Oregon R (Lindsley \& Zimm, 1992), 2b (Pasyukova \& Nuzhdin, 1993), and 98 recombinant inbred (RI) lines derived from these parental lines. Legs were mounted on tape and teeth were counted on the monitor image from a Zeiss Axioplan 2 microscope (the microscope magnification was 100, with another 2-7 magnification from the optovar device connected between the microscope and the camera).

The origin and genotyping of RI lines was described by Nuzhdin et al. (1997). Briefly, the $\mathrm{F}_{1}$ progeny of the parental lines were backcrossed to $2 \mathrm{~b}$, and the backcrossed progeny were randomly mated for four generations. At generation five, 200 individual pairs were selected, and their progeny were inbred by full-sib mating for 25 generations. The genetic constitution of each RI line was previously determined by the analysis of 92 roo TE polymorphic markers that are fixed within the parental lines but segregate between them. The markers were in $1 \mathrm{~B}, 3 \mathrm{E}, 4 \mathrm{~F}, 5 \mathrm{D}, 6 \mathrm{E}, 7 \mathrm{D}, 7 \mathrm{E}, 9 \mathrm{~A}, 10 \mathrm{D}$, $11 \mathrm{C}, 11 \mathrm{D}, 12 \mathrm{E}, 14 \mathrm{C}, 15 \mathrm{~A}, 15 \mathrm{~B}, 15 \mathrm{~F}, 16 \mathrm{~A}, 16 \mathrm{D}, 17 \mathrm{C}$, 18A, 19A, 19C, 21E, 22F, 26F, 29F, 30A, 30D, 33E, 34EF, 35BC, 38A, 38E, 39A, 43A, 43E, 44C, 44F, 46A, $46 \mathrm{C}, 48 \mathrm{D}, 49 \mathrm{D}, 50 \mathrm{~B}, 50 \mathrm{D}, 50 \mathrm{~F}, 57 \mathrm{C}, 57 \mathrm{D}, 57 \mathrm{~F}, 60 \mathrm{E}$, 61A, 61D, 63A, 65A, 65D, 67D, 68B, 68C, 69D, 70C, 71E, 72A, 73D, 76A, 76B, 77A, 77E, 78D, 79C, 79E, $82 \mathrm{D}, 85 \mathrm{~A}, 85 \mathrm{~F}, 87 \mathrm{~B}, 87 \mathrm{E}, 87 \mathrm{~F}, 88 \mathrm{E}, 89 \mathrm{~B}, 91 \mathrm{~A}, 91 \mathrm{D}$, 92A, 93A, 93B, 94D, 96A, 96F, 97D, 97E, 98A, 99A, 99B, 99E and 100A. Chromosome 4 was marked with $s p a^{p o l}$. The lines surviving inbreeding were maintained by mass matings of $\approx 20$ pairs for $\approx 40$ generations before the measurements of the sex comb tooth number were conducted.

The analysis of variance was performed using the GLM and VARCOMP procedures in SAS (SAS, 1988). Composite interval mapping (Zeng, 1994), as implemented by the QTL Cartographer software (Basten 
et al., 1999; < ftp://esssjp.stat.ncsu.edu/pub/qtlcart/> ) was used to test the hypothesis that an interval flanked by two adjacent markers contains a QTL affecting the trait, while simultaneously controlling for the effects of markers situated outside the tested interval (for explanations, refer to Lynch \& Walsh, 1997). Seventy-six unlinked cytological markers were used for the analysis with the parameters 6 (model), and 5 (window size). The conditioning markers were automatically chosen by stepwise forward-backward regression. The likelihood ratio test statistic, $L R$, is $-2 \ln \left(L_{0} / L_{1}\right)$, where $L_{0} / L_{1}$ is the ratio of the likelihood under the null hypothesis (there is no QTL in the interval) to the alternative hypothesis (there is a QTL in the interval). An empirical distribution of the $L R$ test statistics under the null hypothesis of no association between any of the intervals and trait values was obtained by randomly permuting the trait data 1000 times and calculating the maximum $L R$ statistics across all intervals for each permutation. $L R$ statistics from the original data that were exceeded fewer than 50 times by the maximum $L R$ statistics from the permutation are significant at $P=0.05$ (see Doerge et al., 1997; Manly \& Olson, 1999, for discussion of limitations of this analysis).

\section{Results and discussion}

The sex comb tooth number per leg scored 10.5 in Oregon $\mathrm{R}$ and 9.1 in $2 \mathrm{~b}$ lines, with the difference being significant $\left(F_{1,78}=31.9 ; P<0.0001\right)$. To identify QTLs accounting for this intraspecific variability in sex comb tooth number, we screened sex combs on each leg of 40 males from each RI line. The line component of the tooth number variance $(13.4 \%)$ was significant again $\left(F_{97,3822}=14.89 ; P<0.0001\right)$. Neither side (left vs. right leg, fixed effect), nor side by line interaction (random effect) significantly contributed to the variance.

There were two regions of the D. melanogaster $\mathrm{X}$ chromosome for which $L R$ at each marker exceeded the threshold value 11.58 (Fig. 1). One QTL between markers $3 \mathrm{E}$ and $6 \mathrm{E}$ accounted for -0.23 teeth, and another between markers 12E and 19 A accounted for 0.34 teeth. These QTL positions should be taken with caution (Zeng, 1994). The joint effect of QTLs (0.11 teeth) explained only $8 \%$ of the difference between the parental lines in tooth number. To explain this pattern we suggest that there is a large number of small-effect sex comb QTLs segregating between the Oregon $\mathrm{R}$ and $2 \mathrm{~b}$ lines, which we are not able to detect because of the limited power of our analysis.
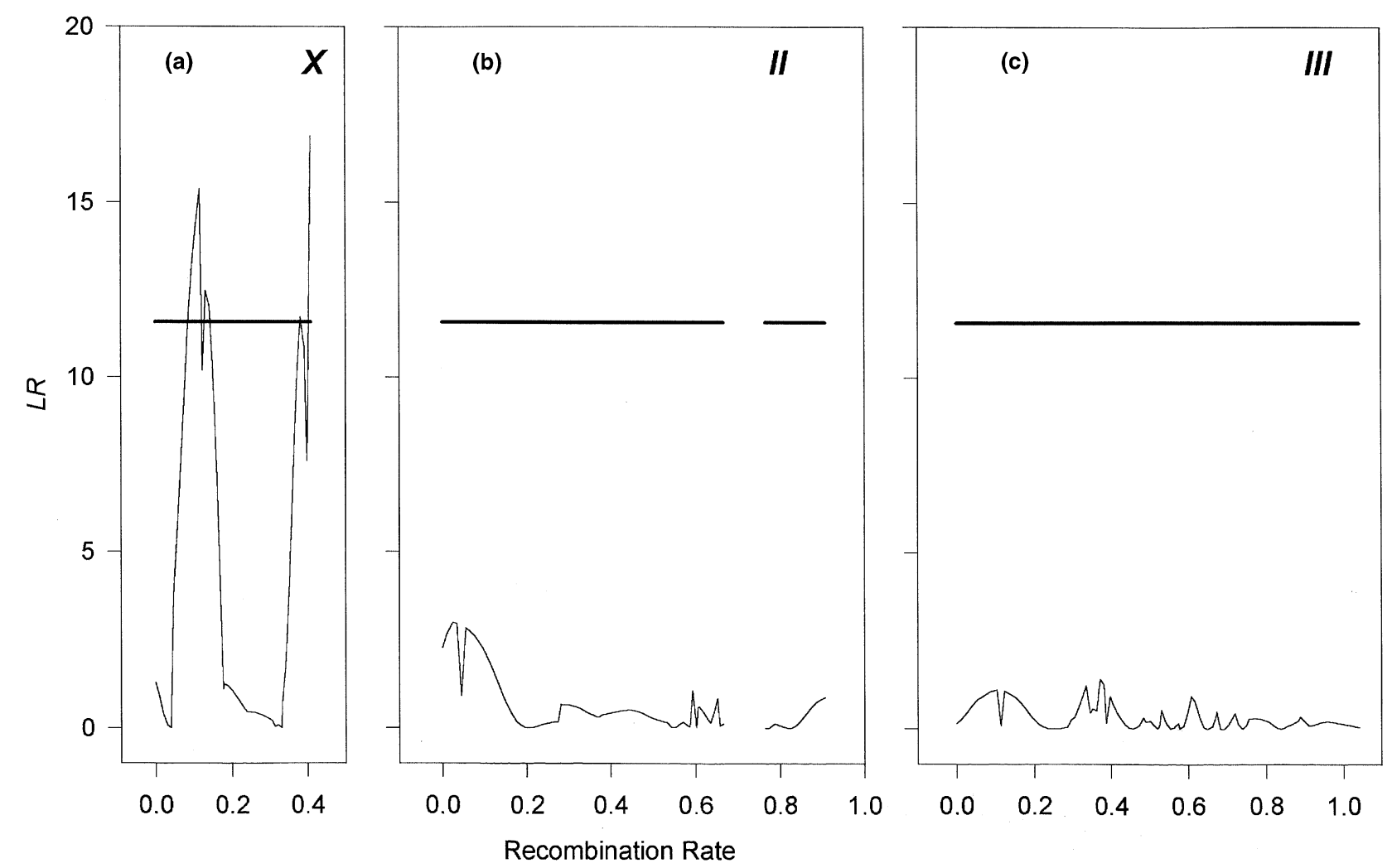

Fig. 1 The intraspecific sex comb QTLs. Panels (a-c) represent three major Drosophila chromosomes (X, 2, and 3). Lines depict likelihood profiles $(\log$ likelihood ratio $-L R$ ). Horizontal lines show statistic thresholds for $P=0.05$.

(C) The Genetical Society of Great Britain, Heredity, 84, 97-102. 
In the analyses of quantitative variation for abdominal and sternopleural bristle number, segregation of a few major-effect chromosome regions frequently explained most of the variance (see Gurganus et al., 1998 for a recent example and citations to earlier papers). However, the intervals in which these QTLs resided were rather large, approaching $1 / 20$ of the genome. It is estimated that there are approximately 20000 genes in Caenorhabditis elegans and, perhaps, the same in Drosophila. Thus, the QTL support intervals typically include 1000 genes, leaving unresolved the issue of whether there were a few factors with large effects or multiple linked factors with small effects within the interval (Lynch \& Walsh, 1998).

For instance, Long et al. (1995) mapped a QTL to the region 89D-92E, which was responsible for 3.6 and 2.4 abdominal bristles, and 1.2 and 1.6 sternopleural bristles in males and females, respectively. Long et al. (1998) associated bristle number with molecular polymorphisms segregating in Delta and Hairless, candidate genes in the bristle formation pathway situated in the mapped interval. Two identified polymorphisms were specific to either abdominal or sternopleural bristles and had much smaller effects than the effects of QTL alleles. Although the QTL mapping experiment may have overestimated, and association studies underestimated, these allelic effects (Long et al., 1998), the discrepancy could also be explained if there were many mutations in different genes of the region 89D-92E. The most thoroughly studied story - molecular genetic dissection of quantitative variability of $A d h$ expression - points to the latter explanation. Indeed, Stam \& Laurie (1996) found that many segregating genes contributed to the variability of $A d h$ activity. Moreover, differences in activity between slow and fast $A d h$ alleles were accounted for primarily by interactions of many nucleotide substitutions within the $A d h$ locus. To infer the distribution of allelic effects for morphological quantitative traits, one clearly needs to identify genes and their polymorphisms instead of chromosomes and their regions.

QTL mapping is, thus, just the first step in inferring the genetic architecture of quantitative traits. The next step is to proceed from QTL to genetic locus. One approach is to search for candidate genes identified by mutations of large effects in the region to which the QTL maps (Mackay, 1995). The biochemical and developmental processes responsible for sex comb formation are among the best understood. Thus, one may hypothesize which genes contribute to interspecific variation in this trait. Most of these genes are in the Polycomb group $(P c-\mathrm{G})$. It has been estimated that there may be as many as $40 P c$-G genes (Landecker et al., 1994). About $15 P c$ $G$ genes have already been described (see Simon, 1995 for review), and more data are likely in the coming years. The $P c-\mathrm{G}$ genes are required for maintaining anterior restriction on the expression of the homeotic selector genes of the $B X-\mathrm{C}$ and Antp-C, and hence for the maintenance of segmental determination during embryonic development (Landecker et al., 1994). Double or triple mutants of $P c-G$ genes have synergistic effects in homeotic transformations compared with single mutations (Messmer et al., 1992). Two smaller groups of sex comb modifiers are represented by genes involved in the terminal stages of leg segmentation, and by genes directing sex-specific expression of somatic cells. A long list of candidate genes for which mutations might be responsible for sex comb variability in nature is available. The positions of some of these candidates match the positions of QTLs found by True et al. (1997) and mapped here (Table 1). Among those, mutations of candidate genes sexcombless and sexlethal seem to be the most promising candidates for future work, as we frequently observed phenotypes intermediate between males and females for the sex comb teeth. Partial rotation of the row and interrupted rows (data are not shown) might be indicative of the weak alleles of these genes.

Sex comb tooth number could, perhaps, be modified by mutations in genes in the bristle formation pathway (see Mackay, 1995, for a review ) because sex comb teeth are modified bristles. True et al. (1997) found the largest effect QTL in the region 73A-84BD. This region also affected anal plate bristle number, clasper bristle number and the number of bristles on the 5 th sternite (True et al., 1997). The RI lines we employed here were previously used to map QTLs for the number of sternopleural and abdominal bristles (Gurganus et al., 1998). The chromosome interval 3E-6E influencing sex combs, affected also the number of sternopleural but not abdominal bristles. In both cases, either pleiotropic effects of a single gene involved in a bristle formation pathway (or sex-specific cell fate pathway), or linkage of genes affecting different traits might be responsible. Although single mutations affecting one bristle trait frequently affect other traits as well (Lyman et al., 1996), most bristle genes have effects limited to very specific patterns (Garcia-Bellido, 1979). Finer-scale genetic dissection is required to choose between these explanations.

Here, we started to dissect the genetics of intraspecific variability in sex combs to test whether standing variation in this quantitative trait serves as a substrate for selection response during speciation. The same chromosome region 12E-19A contributed to both intraspecific variability in $D$. melanogaster and interspecific differences between D. mauritiana and D. simulans. Whether or not these effects can be attributed to the same genes requires further examination. 
Table 1 Candidate genes for sex comb tooth number in Drosophila melanogaster

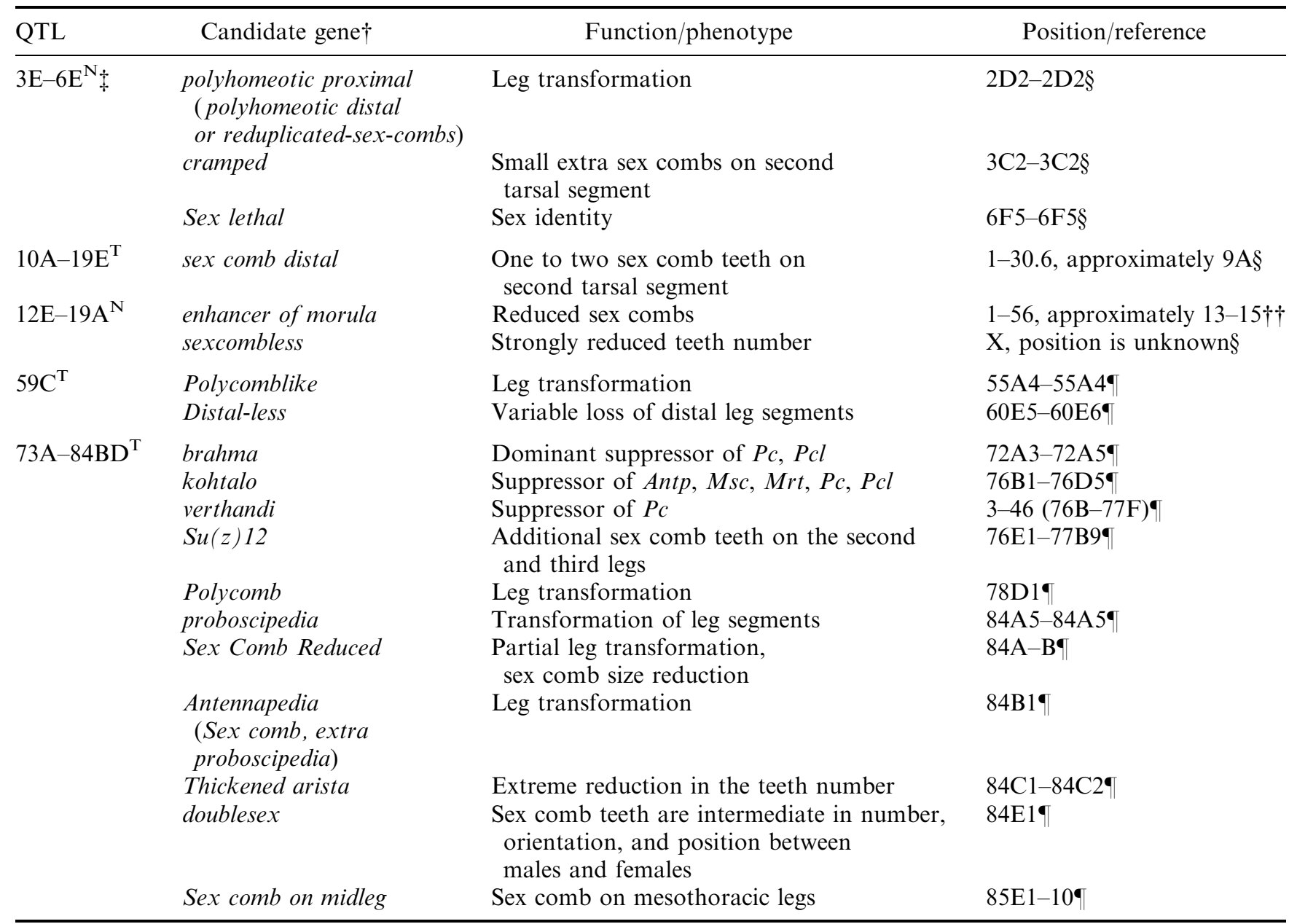

$\uparrow$ Minute mutations usually affecting sex comb teeth number are not listed.

$\ddagger \mathrm{T}$ and $\mathrm{N}$ mark candidate genes mutations in which might correspond to QTLs mapped by True et al. (1997), or by those mapped here. References: §Lindsley \& Zimm (1992); - Saget et al. (1998); ††Reed (1992).

\section{Acknowledgements}

We thank Teresa Leonardo, Chuck Langley, and reviewers for comments on the manuscript. This research was partly supported by UC Davis start up fund, and by a National Science Foundation grant to SVN.

\section{References}

BASTEN, C. J., WEIR, B. S. AND ZENG, Z.-B. 1999. QTL Cartographer, version 1.13. Department of Statistics, North Carolina State University, Raleigh, NC.

CARRoll, S. B. 1995. Homeotic genes and the evolution of arthropods and chordates. Nature, 376, 479-485.

CARSON, H. L. AND LANDER, R. 1984. Inheritance of a secondary sexual character in Drosophila silvestris. Proc. Natl. Acad. Sci. U.S.A., 81, 6904-6907.

(C) The Genetical Society of Great Britain, Heredity, 84, 97-102.
CHOI, Y. H., KWON, Y. W., YOO, M. A. AND LEE, W. H. 1994. Comparative study of morphology on male sex comb variation of the Drosophila species. Korean J. Appl. Entomol., 33, 216-224.

COYNE, J. A. 1985. Genetic studies of three sibling species of Drosophila with relationship to theories of speciation. Genet. Res., 46, 169-192.

COYNE, J. A. AND ORR, H. A. 1998. The evolutionary genetics of speciation. Proc. R. Soc. B, 353, 287-305.

DOERGE, R. W., ZENG, Z.-B. AND WEIR, B. S. 1997. Statistical issues in the search for genes affecting quantitative traits in experimental populations. Stat. Sci., 12, 195-219.

GARCIA-BELLIDO, A. 1979. Genetic analysis of the achaetascute system of Drosophila melanogaster. Genetics, 91, 491-520.

GURGANUS, M. C., FRY, J. D., NUZHDIN, S. V., PASYUKOVA, E. G., LYMAN, R. F. AND MACKAY, T. F. C. 1998. Genotype-environment interaction for quantitative trait loci affecting sensory 
bristle number in Drosophila melanogaster. Genetics, 149, 1883-1898.

KONDRASHOV, A. S. AND KONDRASHOV, F. A. 1999. Interactions among quantitative traits in the course of sympatric speciation. Nature, 400, 351-354.

LANDECKER, H. L., SINClAiRE, D. A. R. AND BLOCK, H. W. 1994. A screen for enhancers of Polycomb and Polycomblike in Drosophila melanogaster. Dev. Genet., 15, 425-434.

LINDSLEY, D. L. AND ZIMM, G. G. 1992. The Genome of Drosophila melanogaster. Academic Press, San Diego, CA.

LONG, A. D., MUllaNeY, S. L., REID, L. A., FRY, J. D., LANGLEY, C. H. AND MACKAY, T. F. C. 1995. High resolution mapping of genetic factors affecting abdominal bristle number in Drosophila melanogaster. Genetics, 139, 1273-1291.

LONG, A. D., LYMAN, R. F., LANGLEY, C. H. AND MACKAY, T. F. C. 1998. Two sites in the Delta gene region contribute to naturally occurring variation in bristle number in Drosophila melanogaster. Genetics, 149, 999-1017.

LYMAN, R. F., LAWRENCE, S. V., NUZHDIN, S. V. AND MACKAY, T. F. C. 1996. Effects of single $P$ element insertions on bristle number and viability in Drosophila melanogaster. Genetics, 143, 277-292.

LYNCH, M. AND WALSH, B. 1998. Genetics and Analysis of Quantitative Traits. Sinauer Associates, Sunderland, MA.

MACKAY, T. F. C. 1995. The genetic basis of quantitative variation: numbers of sensory bristles of Drosophila melanogaster as a model system. Trends Genet., 11, 464-470.

MANLY, K. F. AND OLSON, J. M. 1999. Overview of QTL mapping software and introduction to Map Manager QT. Mamm. Genome, 10, 327-334.

MARKow, T. A., BUSTOZ, D. AND PITNICK, S. 1996. Sexual selection and a secondary sexual character in two Drosophila species. Anim. Behav., 52, 759-766.

MESSMER, S., FRANKe, A. AND PARO, R. 1992. Analysis of the functional role of the Polycomb chromo domain in Drosophila melanogaster. Genes Dev., 6, 1241-1254.
NUZHDIN, S. V., PASYUKOVA, E. G., DILDA, C. A., ZENG, Z.-B. AND MACKAY, T. F. C. 1997. Sex-specific quantitative trait loci affecting longevity in Drosophila melanogaster. Proc. Natl. Acad. Sci. U.S.A., 94, 9734-9739.

PASYUKOVA, E. G. AND NUZHDIN, S. v. 1993. Doc and copia instability in an isogenic Drosophila melanogaster stock. Mol. Gen. Genet., 240, 302-306.

REED, B. 1992. The Genetic Analysis of Endoreduplication in Drosophila melanogaster. PhD Thesis, University of Cambridge.

ROGERS, B. T., PETERSON, M. D. AND KAUfMAN, T. C. 1995. Evolution of the insect body plan as revealed by the Sex combs reduced expression pattern. Development, 124, 149-157.

SAGET, O. F., FORQUignON, F., SANTAMARIA, P. AND RANDSHOLT, N. B. 1998. Needs and targets for the multi sex combs gene product in Drosophila melanogaster. Genetics, 149, 1823-1838.

SAS 1998. SAS/STAT User's Guide. Release 6.03 Edition. SAS Institute Inc., Cary, NC.

SimON, J. 1995. Locking in stable states of gene expression: transcriptional control during Drosophila development. Curr. Opin. Cell Biol., 7, 493-505.

SPIETH, H. T. 1952. Mating behavior within the genus Drosophila (Diptera). Bull. Am. Mus. Nat. Hist., 99, 395-474.

STAM, L. F. AND LAURIE, C. C. 1996. Molecular dissection of a major gene effect on a quantitative trait: the level of alcohol dehydrogenase expression in Drosophila melanogaster. Genetics, 144, 1559-1564.

TRUe, J. R., LIU, J., STAM, L. F., ZENG, Z.-B. AND LAURIE, C. C. 1997. Quantitative genetic analysis of divergence in male secondary sexual traits between Drosophila simulans and Drosophila mauritiana. Evolution, 51, 816-832.

ZENG, Z.-B. 1994. Precision mapping of quantitative trait loci. Genetics, 136, 1457-1468. 